\title{
Prosthetic Rehabilitation of Patient with Ocular Defect using Acrylic Stock Eyes
}

\author{
CJ Venkatakrishnan, Ashish R Jain, R Pradeep, Jacob Mathew Philip, M Narasimman
}

\section{ABSTRACT}

The science and art employed in the profession of dentistry can play an important role in the fabrication of an orbital prosthesis. Anophthalmia (the loss of an eye) not only impairs patient's vision but also create a noticeable deformity on facial appearance and esthetics. The condition is a psychological distress for the patient interrupting his routine work. A prosthodontist as an integral member of the craniofacial rehabilitation team can help raise the spirits and ease the mind of the affected. Mutilation of a portion of a face can cause a heavy impact on the self image and personality of an individual. Acceptable cosmetic results usually can be obtained with a orbital prosthesis. This paper describes prosthetic rehabilitation of a 30 -year-old female patient having a left and right ocular defect. A technique to modify stock eye with heat polymerizing polymethyl methacrylate is illustrated. The resultant prosthesis was structurally durable and esthetically acceptable with satisfactory retention. The importance of meticulous treatment planning to tackle the challenges faced in fabricating an ocular prosthesis is explained with the relevant literature.

Keywords: Anophthalmia, Impression of socket, Prefabricated eye/stock acrylic eyes, Esthetics, Ocular prosthesis.

How to cite this article: Venkatakrishnan CJ, Jain AR, Pradeep R, Philip JM, Narasimman M. Prosthetic Rehabilitation of Patient with Ocular Defect using Acrylic Stock Eyes. Int J Prosthodont Restor Dent 2013;3(4):143-147.

Source of support: Nil

Conflict of interest: None

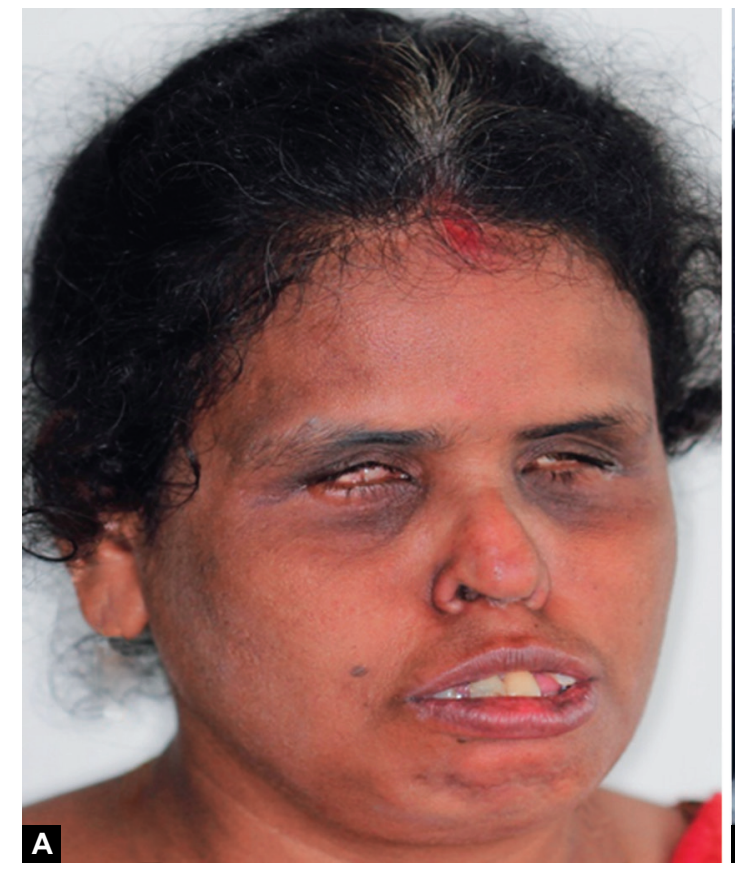

\section{INTRODUCTION}

Eyes are generally the first feature of the face to be noticed. Eye is a vital organ not only in terms of vision but also being an important component of facial expression. Loss of eye has a psychological effect on the patient. So a prosthesis should be provided as soon as possible for the psychological well being of the patient. ${ }^{1}$

Fabrication of an ocular prosthesis can be dated back to Ambrose Pare's gold or silver and glass eye. ${ }^{2}$ These were popular until World War II, till Acrylic resin completely replaced glass eyes. Advantages which gave an upper hand to acrylic resin-prosthesis is solid, light weight, easy to fit and adjust, unbreakable, translucent, easily fabricated, has intrinsic and extrinsic coloring capabilities, increased motility of the prostheses and are inert to the socket secretions.

An ocular prosthesis can be either ready-made (stock) or custom-made. Stock prosthesis comes in standard sizes, shapes and colors. Stock ocular eye requires no special skills or materials for fabrication. They are relatively inexpensive and the entire process is also less time consuming. However, a custom prosthesis is more expensive than a stock prosthesis, and several steps are required for its fabrication. ${ }^{2}$ Before starting the design of the prosthesis, it is essential to assess the psychological component in order to gain the

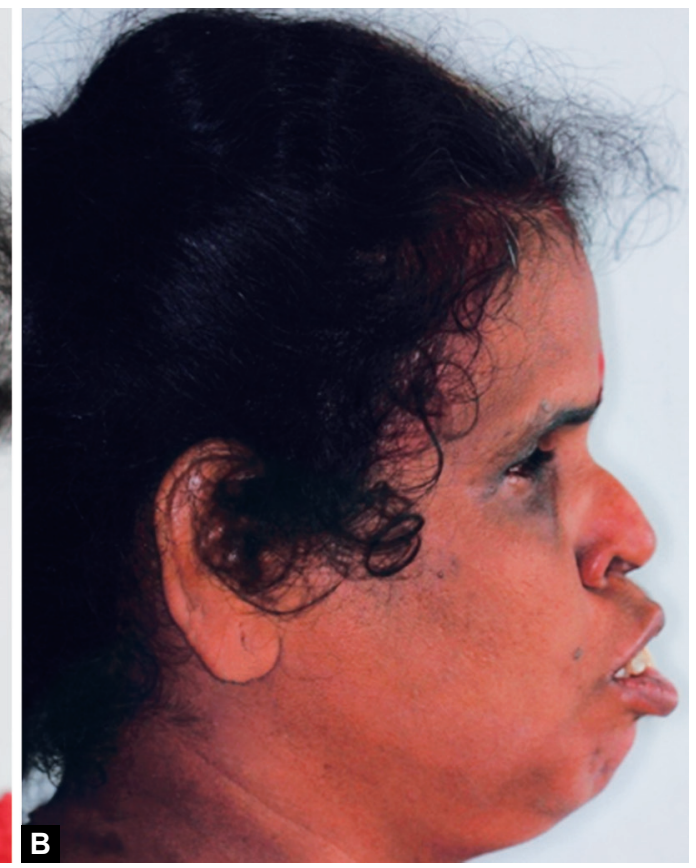

Figs 1A and B: Preoperative photographs (A) frontal view (B) side view 
confidence of the patient, in addition to a detailed medical history that includes the condition that led to the excision and enucleation in order to alert the possibility of recurrence. ${ }^{3}$

The main objective of maxillofacial rehabilitation is to mimic nature. This article attempts to simplify the fabrication of ocular prosthesis by means of modified acrylic stock eyes to bring in natural esthetics.

\section{CASE REPORT}

A 30 years old female patient was referred to the department of prosthodontics for an ocular prosthesis and unesthetic appearance in upper front teeth region of jaw. Intraoral examination revealed that she was undergoing orthodontic treatment, because of longer duration and multiple appointments she was not willing to continue the orthodontic treatment (Figs 1A and B). She presented with severe proclination of maxillary central and lateral incisor, congenitally missing right maxillary canine, grossly decayed right premolars and retained decidous left canine (Figs 2A and $\mathrm{B})$. History revealed that the patient's right and left eyes were enucleated in younger age because of higher bilirubin levels which affected her eyes. On examination of the patient mucosa was healthy. All the movements of the eye socket were found to be favorable for prosthesis. Sulcus depth was sufficient enough to retain the restoration.

\section{TREATMENT PLAN}

It was then decided to extract $11,12,13,14,15,21,22$, 23 with alveoloplasty, followed by fabrication of gum fit prosthesis and later replacement with implant or fixed partial denture (Fig 3). After careful examination of the area of defect, modified stock made ocular prosthesis would be the best to meet her esthetics needs. Patient was explained about the procedure and its limitations.

\section{Choosing the Stock Eye and Subsequent Procedures}

Main objective is to combine the esthetics of stock eyes and the functional adaptation of custom ocular prosthesis for better result and comfort of patient. A stock eye was selected to match the size of ocular defect. For making impression of the enucleated socket, petroleum jelly was applied to the eyebrows and skin around to prevent impression material from sticking to eyelashes. An inlay wax stick (1 cm long and 3-4 mm wide) was attached to the midpoint of the pupil on this artificial eye. Care was taken to center its axis superoinferiorly and mediolaterally. The borders of the artificial eye were reduced to avoid impingement of tissue bed and were made 2 to $3 \mathrm{~mm}$ short of the borders, to allow impression material to flow properly (Fig. 4).

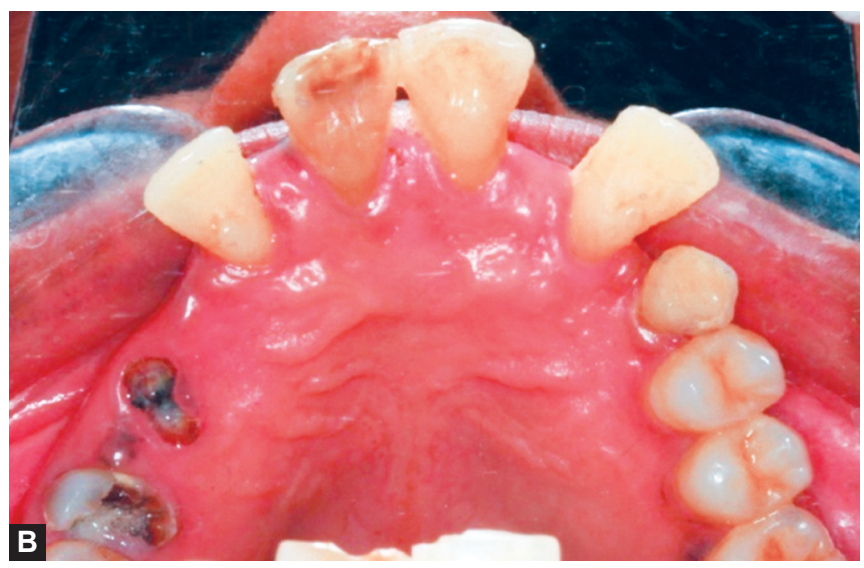

Figs $2 A$ and B: Intraoral photograph of a patient $(A)$ frontal view $(B)$ maxillary occlusal view
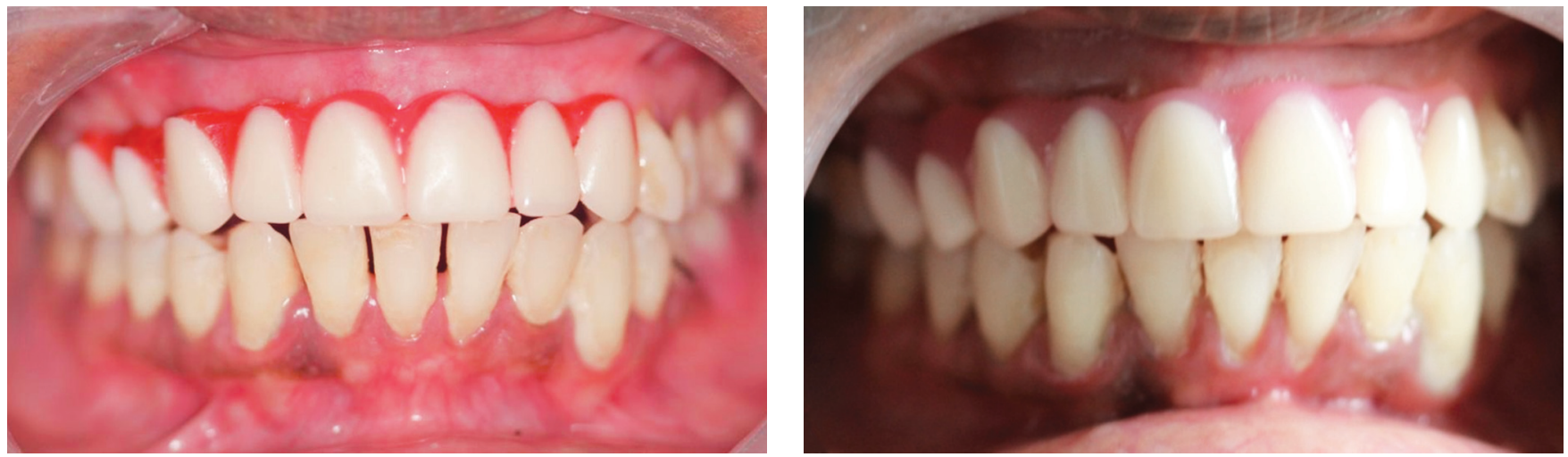

Fig 3: Wax trial and insertion of gum fit denture 

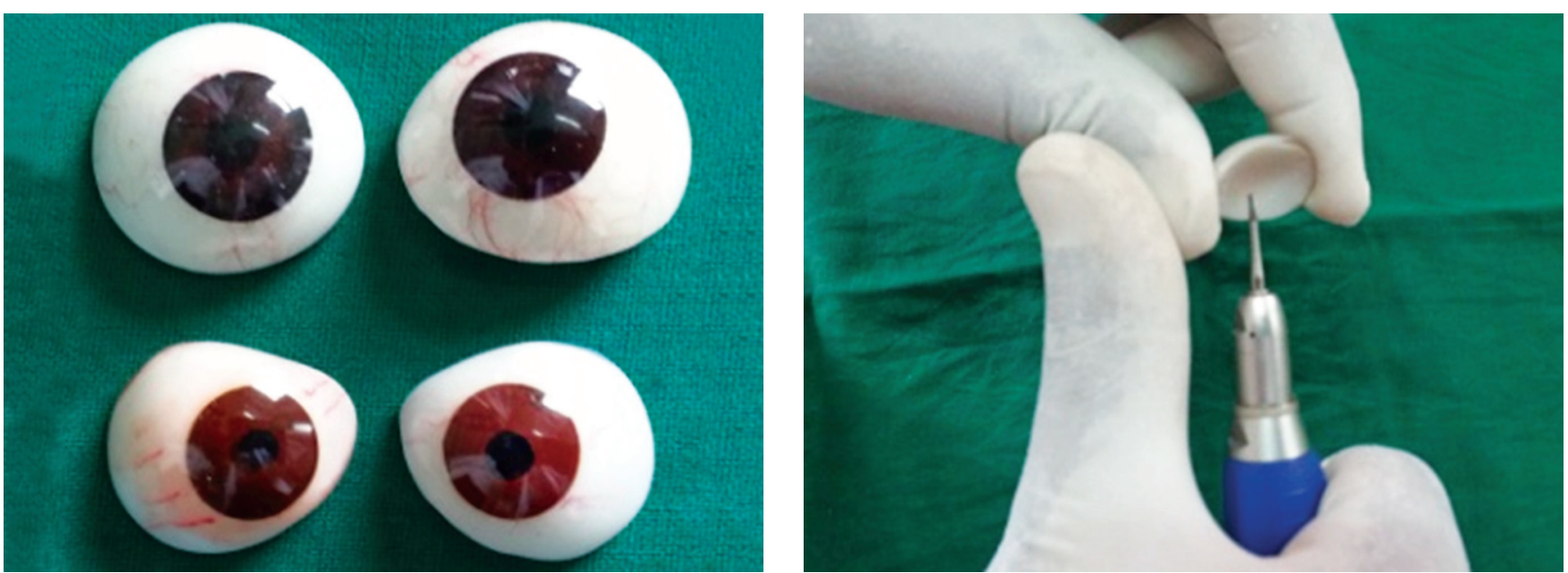

Fig. 4: Modification of acrylic stock eyes
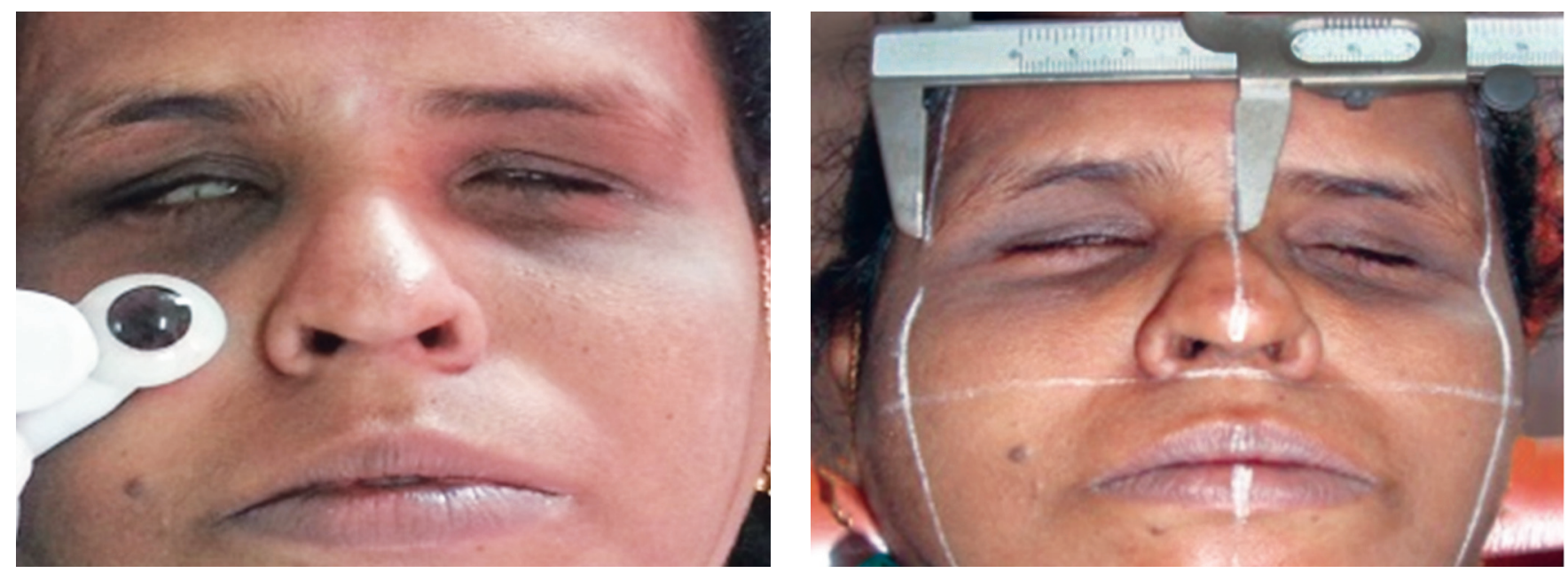

Fig. 5: Facial measurements for orientation of acrylic stock eyes
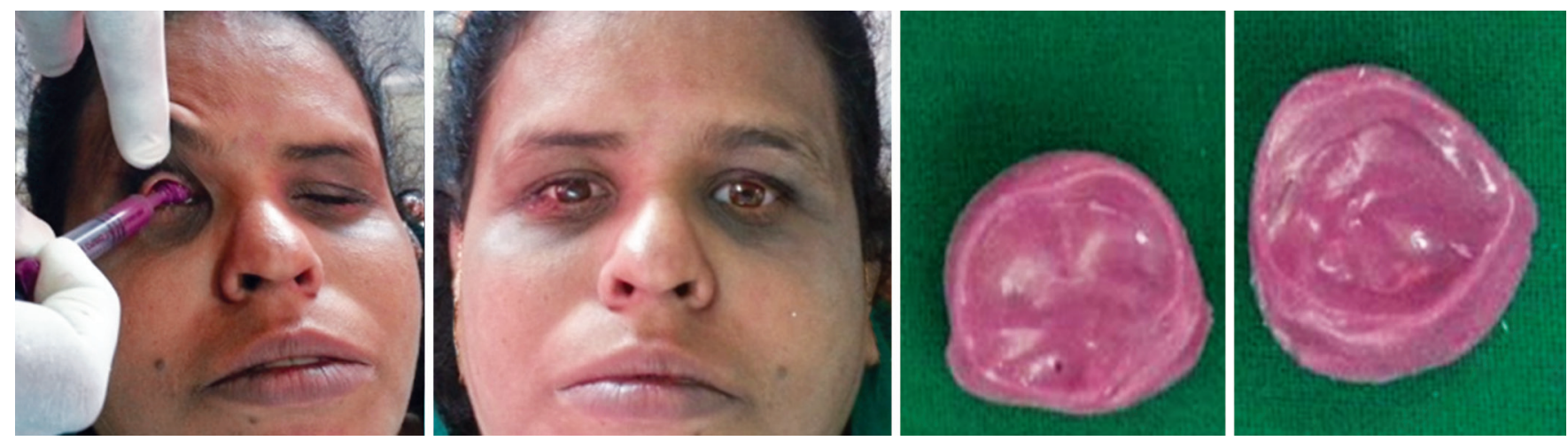

Fig. 6: Ocular impressions

\section{IMPRESSION PROCEDURE}

Medium body addition silicone impression material (Aquasil Lv Ultra, Smart Wetting Impression Material, Dentsply, Detrey Gmbh, Konstanz, Germany) was injected into the socket in little excess to ooze out; while an assistant retracted the upper and lower lids the modified stock eye carrying some material was pressed onto the defect. The patient was instructed to do all the movements of the eyeball as soon as possible and is asked to stare straight at a distant point or object. Impression was removed on set and checked to ensure that all the surfaces were recorded. Erect posture of patient was maintained during the above step. Facial measurements were made using vernier caliper measurements were made to check the position of iris and pupil (Figs 5 and 6).

\section{Prosthesis Fabrication}

Impression was invested in a two-piece crown and bridge flask with laboratory putty (Sil-Tech Putty, Ivoclar Vivadent 

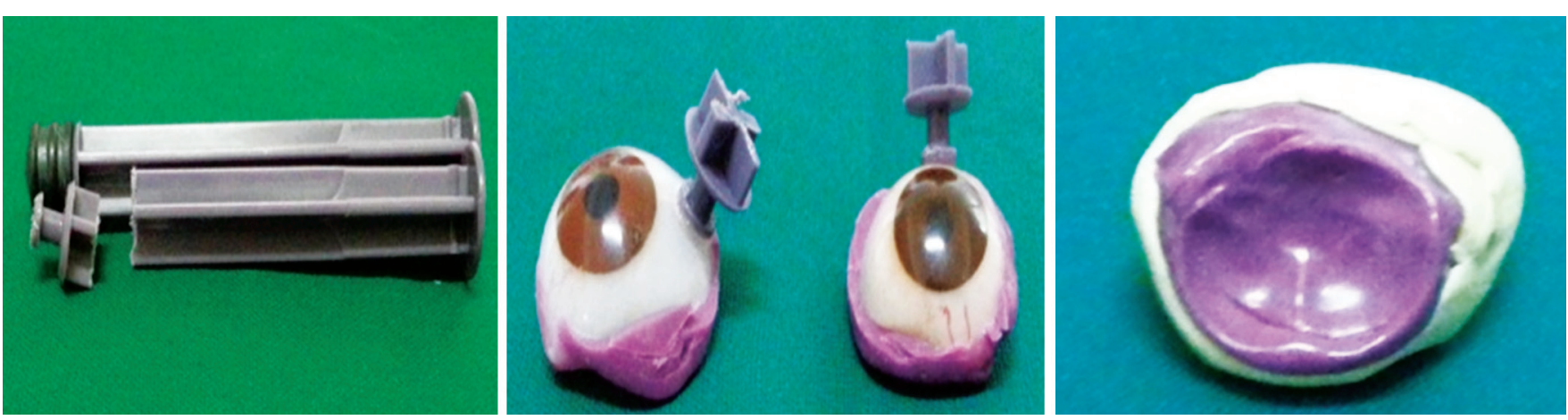

Fig. 7: Laboratory steps for fabrication of ocular prosthesis
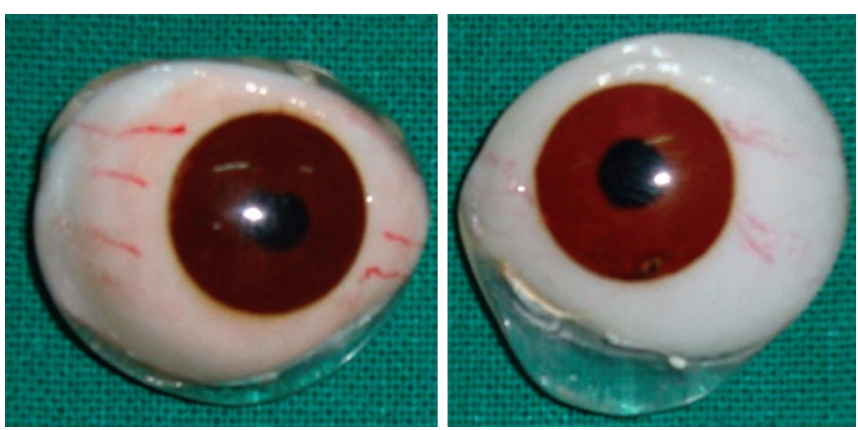

Fig. 8: Finished ocular prosthesis

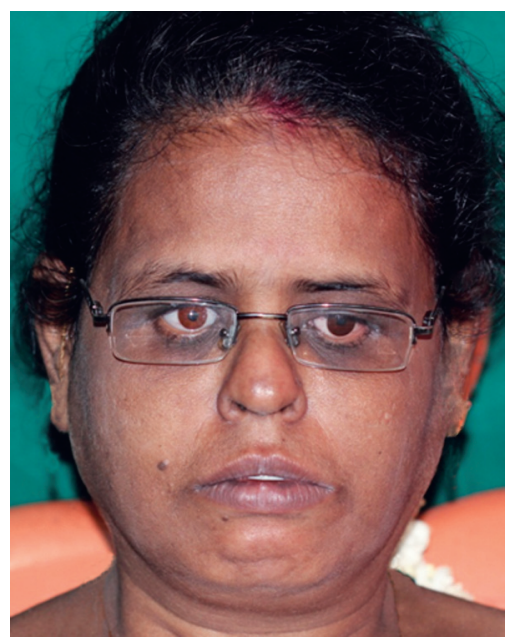

Fig. 9: Postoperative photograph

AG)) and dental stone (Ultrarock, Kalabhai Karson Pvt Ltd, Mumbai, India) and processed conventionally using selected heat cured tooth colored acrylic resin. Processing included a bench press under 3500 psi pressure for at least 1 hour followed by slow curing cycle. The processed prosthesis was deflasked and polished (Figs 7 and 8). Prior to insertion the polished prosthesis was disinfected in a solution of $0.5 \%$ chlorhexidine and $70 \%$ isopropyl alcohol for 5 minutes and then rinsed in sterile saline solution to avoid chemical irritation. Prosthesis was inserted into the socket and evaluated in appearance and location after 10 minutes to allow protective blepharospasms of the orbicular muscles to subside (Fig. 9). Small discrepancies were adjusted by grinding the posterior and peripheral surfaces of the prostheses, so as to achieve similar position as contralateral eye on straight gaze and later polished.

\section{INSTRUCTIONS TO THE PATIENTS}

The patient was taught the proper method of removal and insertion. The patient was instructed to wear the prosthesis all day, but to remove and wash it with a mild soap once a day. A follow-up was done on the 4th day to check for signs of irritation of the socket and relieve the prostheses as needed. During recall visit the fit, mobility, and direction of gaze of the prosthesis should be first checked. Next the prosthesis must be removed and checked for scratches, chips, bacterial growth and accretions, etc. If the prosthesis is not too old, not contaminated with bacteria, and not chipped or deeply scratched, then careful repolishing can be done to remove scratches found due to dust and dirt. Only those compounds specifically prescribed for eye prosthesis should be used. The patient was then instructed to visit at 6 months intervals or as needed.

\section{DISCUSSION}

The esthetic and psychological benefits of an ocular prosthesis have motivated a constant research in improvement of its prosthetic technique. The general consensus among authors is that close matching the natural eye is the key to mask the loss and achieve an esthetic outcome for patients with ocular defect. The ocular prosthesis is an artificial replacement for the bulb of the eye. After the surgeon enucleates the eye, prosthodontist or anaplastologist is a person who comes into an act of providing the patient with an artificial eye to overcome the agony of losing an eye. A well-made and properly made ocular prosthesis maintains its orientation when patient performs various movements. With the advent of newer materials like heat cure acrylic resin (DPI) as used here, it is possible to fabricate prosthesis with a life-like appearance. $^{4}$

The literature has suggested many techniques for the fabrication of ocular prosthesis. Stock eye prosthesis was 
advocated by Laney. ${ }^{5}$ These prefabricated ocular prostheses made of glass or acrylic resin are known as 'stock eyes' that come in standard sizes, shapes and colors. These are relatively inexpensive and can be delivered quickly.

\section{Need to Modify/Customize a Stock Eye}

Customizing such a stock eye improves adaptation to the underlying tissues, increases mobility of the prostheses and improves facial contours. Moreover the use of stock ocular prosthesis of appropriate size and color cannot be neglected; as it provide better results functionally as well as esthetically. It retains shape of defective socket, prevents collapse of lids, provides muscular functions of the lids, maintains palpebral opening, and gives a gaze similar to that of natural eye, also it helps to reduce the incidence of conjunctival abrasion or ulceration. It also enhances tissue health by reducing potential stagnation spaces at the prosthetic tissue interface. Fluid collection in the space could cause tissue irritation and increase bacterial growth. Using implants can enhance the prosthetic rehabilitation which can co-ordinate the movements with natural eye, but not always possible and feasible also. ${ }^{6-8}$

Many clinicians have concluded that iris color of prosthetic eye is most important consideration for the esthetic acceptance of the prosthesis. The availability of needed iris color and lack of control over the size of the iris and pupil can be a limitation to the above technique, which can be overcome by using various sizes and colors of prefabricated eyes. ${ }^{9}$ Our case report provides acceptable esthetic results as it closely replicates the patient's skin tone with minimal color adjustments and modifications. A modified stock ocular prosthesis is an excellent alternative, which is relatively inexpensive and easy to fabricate or materials required for its fabrication.

\section{CONCLUSION}

An anophthalmic patient was rehabilitated to an esthetic appeal by customizing a stock eye. This technique allowed the proper recording an impression of the ocular defect tissue bed which in turn increased its movement due to excellent adaptation to the walls of the socket. Most patients can be treated with the modified prefabricated eye technique with excellent results. The procedure used here is simple and cost effective. Although the patient cannot see by this prosthesis, this prosthesis will increase the self confidence of the patient to face the world.

\section{REFERENCES}

1. Park K. Preventive and social medicine. Banarsidas Publishers. 19th ed. 2007.

2. Reis CR, Dias BR, Carvalho MCJ. Evaluation of iris color stability in ocular prosthesis. Braz Dent J 2008:19(4):370-377.

3. Artopoulou LI, Montgomery CP, Wesley JP. Digital imaging in the fabrication of ocular prostheses. J Prosthet Dent 2006:95:327-330.

4. Prasad R, Bhambhani R, Joshi S. Esthetic problem: prosthetic solution for an ocular defect. World J Dent 2012;3(3):269-272.

5. Laney WR, Gardner AF. In: Maxillofacial. Prosthetics. Littleton: PSG Publishing 1979;279-306.

6. Dixit S, Shetty P, Bhat GS. Ocular prosthesis in children clinical report. Kathmandu University Medical Journal 2005;3(1):9: 81-83.

7. Brown K. Fabrication of an ocular prosthesis. J Prosthet Dent 1970;24(2):226-235.

8. Jain S, Makkar S, Gupta S, Bhargava A. Prosthetic rehabilitation of ocular defect using digital photography: a case report. J Indian Prosthodont Soc 2010 July-Sept;10(3):190-193.

9. Appanna PA, Porwal A, Verma N. Impression techniques for ocular prosthesis: a clinical review. International Journal of Dental Clinics 2009;1(1):27-31.

\section{ABOUT THE AUTHORS}

\section{CJ Venkatakrishnan}

Research Scholar, Bharat University; Professor and Head, Department of Prosthodontics, Tagore Dental College and Hospital, Chennai, Tamil Nadu, India

\section{Ashish R Jain (Corresponding Author)}

Senior Lecturer, Department of Prosthodontics, Tagore Dental College and Hospital, Chennai, Tamil Nadu, India, Phone: 09884233423, e-mail: dr.ashishjain_r@yahoo.com

\section{R Pradeep}

Reader, Department of Prosthodontics, Tagore Dental College and Hospital, Chennai, Tamil Nadu, India

\section{Jacob Mathew Philip}

Senior Lecturer, Department of Prosthodontics, Tagore Dental College and Hospital, Chennai, Tamil Nadu, India

\section{Narasimman}

Senior Lecturer, Department of Prosthodontics, Tagore Dental College and Hospital, Chennai, Tamil Nadu, India 